\title{
Commentary on Elective versus therapeutic neck dissection in node-negative oral cancer
}

\section{BACKGROUND}

Lymph node metastasis is the single most important prognostic factor in oral squamous cell carcinoma. ${ }^{[1]}$ With the reported incidence of occult metastasis in early stage (T1, T2) oral cancer of $30 \%$, there are opposing clinical practices. There are proponents for elective neck dissection (END) and others for watchful-wait, and to undertake therapeutic neck dissection (TND) for those who are found to have developed nodal metastasis during follow-up. Though there were four previous randomized trials and a meta-analysis, comparing these two clinical practices, being underpowered and due to methodological errors the issue remains unresolved.

In this practice-changing prospective randomized clinical trial comparing END and TND, D'Cruz et al. ${ }^{[2]}$ has categorically demonstrated the superiority of END in patients with T1, T2, and N0 squamous cell carcinoma of oral cavity in terms of overall survival and disease-specific survival.

\section{TRIAL DESIGN}

The study was designed to accrue 710 patients with clinical $\mathrm{T} 1, \mathrm{~T} 2$, and $\mathrm{N} 0$ oral squamous cell carcinoma. The neck was deemed N0 by physical examination and ultrasound scan. In the END group, the neck was managed by selective neck dissection involving levels $1-3$ and if the nodes prove to be positive by pathology, they were converted to modified radical neck dissection (MRND) covering levels 1-5. In the TND group, those patients who develop nodal relapse were managed by either MRND or radical neck dissection. The decision for adjuvant radiation was left to the discretion of the treating physicians. The post-treatment follow-up was randomized either into physical examination alone or physical examination and ultrasound scan. The study had a stringent follow-up regimen with 4 weeks for first 6 months, followed by 6 weeks for next 6 months, then every 8 weeks for next 12 months, and every 12 weeks thereafter. Between 2004 and 2014, 596 patients were enrolled. A planned interim analysis was carried out on the first 500 patients who have completed at least 9 months of followup (median follow-up 39 months). Based on the results of the analysis of the first 500 patients (245 in the END and 255 in the TND) demonstrating superiority of END, the

\begin{tabular}{|l|l|}
\hline \multicolumn{2}{|c|}{ Access this article online } \\
\hline Quick Response Code: & Website: \\
\hline & www.ijmpo.org \\
\hline
\end{tabular}

data safety monitoring committee stopped the accrual of patients in June 2014. The present article reports the result of this patient group.

\section{PRINCIPAL FINDINGS}

1. END resulted in improvement in overall survival $(80 \%$ vs. $67.5 \% ; P=0.01)$ and disease-specific survival (69.5\% vs. $45.9 \% ; P<0.001)$.

2. There were $81(33.3 \%)$ recurrences in the END and $146(57.7 \%)$ in the TND group.

3. Adjuvant radiation after primary surgery was given in $118(48.6 \%)$ patients in the END and $86(34 \%)$ patients in the TND group. After salvage surgery, 25 (10.3\%) patients in the END and 71 patients $(28.1 \%)$ in the TND group received adjuvant radiation.

4. $25 / 81(30 \%)$ recurrences in the END was in the neck nodes, while that of TND group was 108/146 (74\%). The local recurrence was significantly higher in the END, $23(28 \%)$ compared to $7(4.8 \%)$ in the TND group.

5. $72 / 243(29.6 \%)$ in the END and $114 / 253(45.05 \%)$ in the TND group developed node failures. These were $52.8 \%$ $\mathrm{N} 1,47.2 \% \mathrm{~N} 2 \mathrm{~b} / \mathrm{c}$ in the END and $31.5 \% \mathrm{~N} 1,9.6 \% \mathrm{~N} 2 \mathrm{a}$, $39.4 \% \mathrm{~N} 2 \mathrm{~b} / \mathrm{c}$, and $18.4 \% \mathrm{~N} 3$ in the TND group.

6. While extracapsular spread was observed only in $51.4 \%$ in END, $93.02 \%$ had extracapsular spread in the TND group. $20(17.5 \%)$ neck recurrences in the TND group were deemed to be unresectable at the time of diagnosis.

7. Post-hoc sub-group analysis showed lack of benefit for END in patients with tumor depth of invasion measuring $<3 \mathrm{~mm}(n=71)$.

8. Addition of ultrasound scan to physical examination did not improve early detection of neck failures (data not provided). $21(18.4 \%)$ of the neck recurrences were detected with advanced N3 disease. Of which, $20(17.5 \%)$ were considered unresectable.

\section{STRENGTHS OF THE STUDY}

1. Well-powered and well-designed trial.

2. Single center trial ensuring consistency in imaging, pathology, surgery, and adjuvant radiation.

\footnotetext{
This is an open access article distributed under the terms of the Creative Commons Attribution-NonCommercial-ShareAlike 3.0 License, which allows others to remix, tweak, and build upon the work non-commercially, as long as the author is credited and the new creations are licensed under the identical terms.
}

How to cite this article: Kuriakose MA. Commentary on Elective versus therapeutic neck dissection in node-negative oral cancer. Indian J Med Paediatr Oncol 2015;36:137-9. 
3. Despite the fact that the study was carried out in India, where buccal mucosal cancer is the predominant sub-site, the design of the study ensured weightage to tongue and floor of mouth making the result of study applicable to a global patient population.

4. Large study population allowed sub-group analysis that helped to generate hypothesis for further studies.

5. Undertaking prospective randomized surgical trials are a daunting task. The authors to be congratulated for carrying out a clinically relevant and stringent surgical clinical trial.

\section{DRAWBACKS OF THE STUDY}

1. As demonstrated by the present trial and several previous studies, ${ }^{[3]}$ subjective nature of ultrasound scan offer questionable value in the evaluation of neck. In addition, the study also included patients with indeterminate and suspicious node by ultrasound scan in the trial. The latter patients should not have been considered as N0.

2. The high incidence of node relapse of $114 / 253(45 \%)$ in the TND group as opposed to $72 / 243(29.6 \%)$ in the END group cannot be readily explained. This may have been contributed by the lack of sensitivity of initial neck evaluation by ultrasound scan. As computed tomography scan is now routinely used for both primary and neck evaluation, use of this modality could have avoided this error. Higher proportion of patients in the END group receiving adjuvant radiation could have lowered the neck failure rate.

3. Despite rather stringent follow-up regimen, 21 subjects in the TND group were diagnosed at N3 and 20 of which were deemed inoperable. This further attests the limitation of physical examination for evaluation of neck after radiotherapy and poor sensitivity of ultrasound scan.

4. In the present trial, subjects who were found to have pathological positive node in the END group and those with clinical node relapse in the TND group underwent MRND or RND. As level V is rarely involved in oral cavity cancers and these patients would require adjuvant radiotherapy, a more conservative approach of selective neck dissection would have been equally effective. ${ }^{[4]}$

5. A higher percentage of patients in the END group receiving adjuvant radiotherapy on the basis of nodal disease may have skewed the oncological outcome in favor of the END group.

6. Despite the fact that $93 \%$ of the patients in the TND had extracapsular extension, these patients were denied chemo-radiotherapy as adjuvant treatment, which is now considered as a standard of care. ${ }^{[5]}$

\section{CLINICAL IMPLICATIONS}

The result from this study has established END as a standard of care for the management of occult metastasis in patients with early stage oral squamous cell carcinoma. The sub-group analysis suggests that END will have beneficial effect only in those tumors with a depth of invasion over $3 \mathrm{~mm}$. Though $3 \mathrm{~mm}$ depth is determined by histopathologic examination, one can consider any clinically palpable oral cavity tumors to have depth over $3 \mathrm{~mm}$.

\section{FUTURE PERSPECTIVES}

The management of neck has evolved over the years from radical neck dissection to MRND and the selective neck dissection in an attempt to preserve function without compromising oncological outcome. Dr. D'Cruz trial has clearly demonstrated the need for END to manage N0 neck. Though this trial demonstrated superior oncological outcome compared to watchfulwait policy, one should accept that this is over treatment in about $70 \%$ of the patients who do not have metastatic nodal disease. There are emerging data to suggest that sentinel node biopsy in lieu of END to manage N0 neck disease is equally effective. ${ }^{[6]}$ However, it requires larger prospective trial demonstrating oncologic equivalence to END.

Currently depth of invasion is the only clinically useful marker that can predict risk of node metastasis. With the improved understanding from tumor biology, one may identify more precise molecular markers that could better delineate metastatic markers of primary tumors. ${ }^{[7]}$

\section{CONCLUSION}

The results from this study have established END as a standard of care for T1, T2, and N0 oral squamous cell carcinoma.

\section{Moni Abraham Kuriakose $e^{1,2}$}

${ }^{1}$ Department of Surgical Oncology, Mazumdar Shaw Cancer Center, Bengaluru, Karnataka, India, ${ }^{2}$ Department of Head and Neck, Plastic and Reconstructive Surgery, Roswell Park Cancer Institute, Buffalo, New York, USA.

E-mail: makuriakose@gmail.com

\section{REFERENCES}

1. Amit M, Yen TC, Liao CT, Binenbaum Y, Chaturvedi $P$, Agarwal JP, et al. Clinical nodal stage is a significant predictor of outcome in patients with oral cavity squamous cell carcinoma and pathologically negative neck metastases: Results of the International Consortium for Outcome Research. Ann Surg Oncol 2013;20:3575-81.

2. D'Cruz AK, Vaish R, Kapre N, Dandekar M, Gupta S, Hawaldar R, et al. Elective versus therapeutic neck dissection in node-negative oral cancer. N Engl J Med 2015;373:521-9.

3. Liao LJ, Lo WC, Hsu WL, Wang CT, Lai MS. Detection of cervical lymph node metastasis in head and neck cancer 
patients with clinically NO neck-a meta-analysis comparing different imaging modalities. BMC Cancer 2012;12:236.

4. Battoo AJ, Hedne N, Ahmad SZ, Thankappan K, lyer S, Kuriakose MA. Selective neck dissection is effective in N1/ N2 nodal stage oral cavity squamous cell carcinoma. J Oral Maxillofac Surg 2013;71:636-43.

5. Bernier J, Cooper JS. Chemoradiation after surgery for high-risk head and neck cancer patients: How strong is the evidence? Oncologist 2005;10:215-24.
6. Rigual N, Loree T, Frustino J, Jayaprakash V, Cohan D, Sullivan $\mathrm{M}$, et al. Sentinel node biopsy in lieu of neck dissection for staging oral cancer. JAMA Otolaryngol Head Neck Surg 2013;139:779-82.

7. Takes RP, Rinaldo A, Rodrigo JP, Devaney KO, Fagan JJ, Ferlito A. Can biomarkers play a role in the decision about treatment of the clinically negative neck in patients with head and neck cancer? Head Neck 2008;30:525-38.

\section{“QUICK RESPONSE CODE” LINK FOR FULL TEXT ARTICLES}

The journal issue has a unique new feature for reaching to the journal's website without typing a single letter. Each article on its first page has a "Quick Response Code". Using any mobile or other hand-held device with camera and GPRS/other internet source, one can reach to the full text of that particular article on the journal's website. Start a QR-code reading software (see list of free applications from http://tinyurl.com/yzlh2tc) and point the camera to the QR-code printed in the journal. It will automatically take you to the HTML full text of that article. One can also use a desktop or laptop with web camera for similar functionality. See http://tinyurl.com/2bw7fn3 or http://tinyurl.com/3ysr3me for the free applications. 